\title{
MODELACIÓN FÍSICA EN DIMENSIONES \\ REDUCIDAS DE MUROS DE CONTENCIÓN EN TIERRA ARMADA CON GEOTEXTIL
}

(Physical Modelling in Reduced Dimensions of Contention Walls Made of Reinforced Earth by Geo-Textile)

Jairo Martín Espitia López*, Ana Carolina Ramírez Ruiz**, José Rodrigo Méndez Zuluaga**

*Escuela de Ingeniería de Minas, Grupo de Investigación GEAM, UPTC, jairomartin.espitia@uptc.edu.co

**Escuela de Posgrados, Especialización en Geotecnia Vial, UPTC, ingkarorr@hotmail.com - rodrigomendez2008@yahoo.es

(Recibido Mayo 15 de 2006 y aceptado Abril 23 de 2007)

\section{Resumen:}

Con el objeto de ensayar modelos escalados, se construyó un equipo para la modelación física de muros de contención en tierra reforzada con geotextil. El equipo consiste en un marco de acero prismático con paredes en lámina de acero, una de ellas en acrílico. En la construcción de los modelos se utilizó arena de peña lavada, el material de refuerzo consiste en una tela tipo interlón, material al que se le realizaron los ensayos estándar que se adelantan a los geotextiles en la empresa Pavco. El modelo consiste en un muro de $0,60 \mathrm{~m}$ de alto, $0,80 \mathrm{~m}$ de largo y $0,20 \mathrm{~m}$ de ancho de arena reforzada con tela. En total se realizaron seis modelos con diferentes espesores de capa reforzada; los modelos se sometieron a una carga de compresión y durante el proceso de carga se observó el mecanismo de falla y la curva esfuerzodesplazamiento del modelo.

Palabras clave: Tierra armada, Geotextil, Modelación física, Muros de contención.

\section{Abstract:}

A machine was built with the purpose to try physical modelling by making reinforced earth retaining walls with geo-textile. The machine consists in a prismatic steel frame with steel sheet walls, including one in acrylic. The models was used sand out of washed rock, and as reinforcement was used an especial fabric, which underwent the standard testing in the factory PAVCO. The model consists in a retaining wall, of $0.60 \mathrm{~m}$ high, $0.80 \mathrm{~m}$ long and $0.20 \mathrm{~m}$ wide, full of sand reinforced with the fabric. A total of 6 models were made with different thickness of reinforced layer. The models were subject to a compression load, and during the loading process it was observed the failure mechanism and stress-displacement behavior.

\section{INTRODUCCIÓN}

$\mathbf{E}$ 1 concepto de la tierra reforzada no es nuevo, como se podría pensar; desde hace miles de años se han usado materiales naturales como elementos de refuerzo en las estructuras de tierra construidas por la humanidad. Los adelantos más notables en los métodos de retención se empezaron a observar desde la segunda mitad del siglo pasado, dándose principalmente en los países de Europa occidental, donde tuvieron que iniciar un largo proceso reconstructivo tras la Segunda Guerra Mundial. Fue precisamente esta circunstancia la que originó un gran deseo por descubrir y aplicar nuevas técnicas y métodos de ingeniería; por esta razón se empezaron a ver grandes innovaciones que gradualmente fueron pasando a otros países.

En 1963, el ingeniero y arquitecto francés Henry Vidal patentó el sistema de Tierra Armada, después de observar el mecanismo natural de refuerzo que formaban las agujas de pino sobre la arena de playa (Centeno, 1985). La primera aplicación de tierra armada se dio con refuerzo en tiras de acero galvanizado que se introducía a determinada profundidad en el suelo.
Los geosintéticos fueron introducidos en los primeros años de la década de los setenta del siglo XX como alternativa al sistema de refuerzo con barras de acero (Holtz \& Lee, 2002). Durante esta década se llevaron a cabo muchos experimentos con geotextiles tejidos y no tejidos para la construcción de muros y terraplenes reforzados.

En general, las estructuras reforzadas con geosintéticos son una excelente alternativa para estabilizar taludes y recuperar bancas de vías (López, 1999). Esta técnica aumenta la estabilidad global e interna del muro, debido a que el geosintético brinda confinamiento lateral que, complementado con una compactación eficiente del material, reduce la posibilidad de falla del material.

Son múltiples los beneficios que presenta el uso de muros en tierra armada con geotextil; dentro de ellos se destacan: facilidad de instalación, flexibilidad y tolerancia a las deformaciones, reducción de costos, permiten excelentes posibilidades paisajísticas y estéticas, y presentan excelente desempeño ante eventos sísmicos (Holtz \& Lee, 2002). 
En el país, y a nivel mundial, se ha utilizado la solución de tierra armada con geotextil en innumerables e importantes proyectos; este panorama motiva a los autores a iniciar investigaciones que buscan el entendimiento del mecanismo de falla y el comportamiento esfuerzo-deformación de muros de contención en tierra reforzada con geotextil.

\section{CARACTERIZACIÓN DE MATERIALES}

Para el desarrollo de esta investigación se consideró conveniente utilizar suelos con características homogéneas, que facilitaran la construcción de los modelos, permitieran un proceso de compactación sencillo y no afectaran los resultados por problemas de escala. Como material de refuerzo se tuvo en cuenta el uso de telas que tuvieran características similares al geotextil; para esto se utilizaron tres tipos de telas (velo, tela de dacrón y material de interlón), a las cuales se les practicaron ensayos estándar que se llevan a cabo a los geotextiles; los resultados fueron supervisados por el ingeniero Ramiro Rubio, experto en geosintéticos, quien dio un concepto favorable para usar una de ellas.

\subsection{Suelo}

El suelo utilizado en el proyecto corresponde a arena de peña lavada; presenta un coeficiente de uniformidad $\mathrm{Cu}=2,6 \mathrm{y}$ un coeficiente de curvatura de $\mathrm{Cc}=0,8$, y clasifica como una arena SP de acuerdo con la clasificación USCS. En las figuras 1 y 2 se presenta la curva granulométrica y la curva de compactación del material; la humedad óptima resultó igual a $8,5 \%$, la cual se tomó como parámetro para la construcción de los modelos. En la tabla 1 se muestran los resultados de laboratorio adelantados a la arena.

Tabla 1. Propiedades de la arena.

\begin{tabular}{|c|c|c|c|c|}
\hline Clasificación USCS & IP & $\phi\left(^{(}\right)$ & $\gamma \mathbf{d m a x}(\mathbf{k N} / \mathbf{m} 3)$ & Wóptima (\%) \\
\hline SP & N.P. & 33 & 17.31 & 8.5 \\
\hline
\end{tabular}

Nota: N.P. se refiere a material no plástico; el ángulo de fricción interna se obtuvo a partir de la ejecución de dos pruebas de corte directo.

\subsection{Material de refuerzo}

Como material de refuerzo fue utilizada una tela de interlón, que fue ensayada previamente en Pavco, en conjunto con las otras dos telas consideradas inicialmente. La descripción general de las telas ensayadas se da a continuación (ver tabla 2):

- Interlón: Referencia NF35, blanco plus suave. Es un material con características no tejidas; presenta un comportamiento semejante a un geotextil, según el concepto dado por el ingeniero Ramiro Rubio (Ingeniero de proyectos de la división de Geosistemas-Pavco).

- Velo: de color morado, con referencia muselina 100 poliéster tejido plano.

- Tela de dacrón: de color azul rey, tipo poliéster L 100.

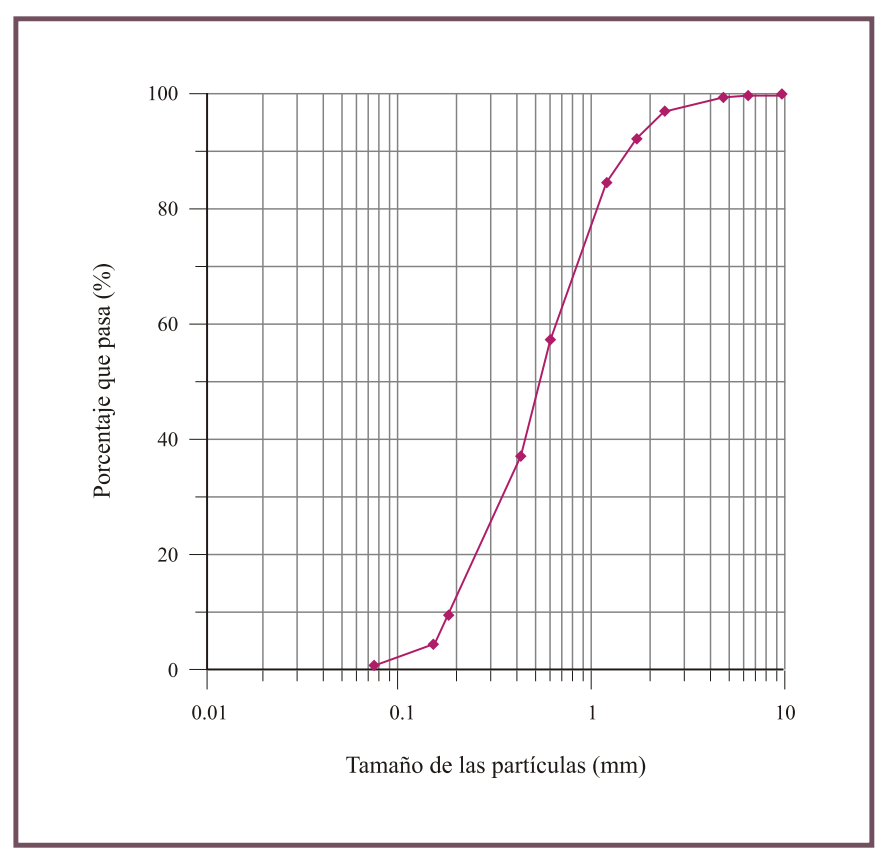

Figura 1. Curva granulométrica de la arena.

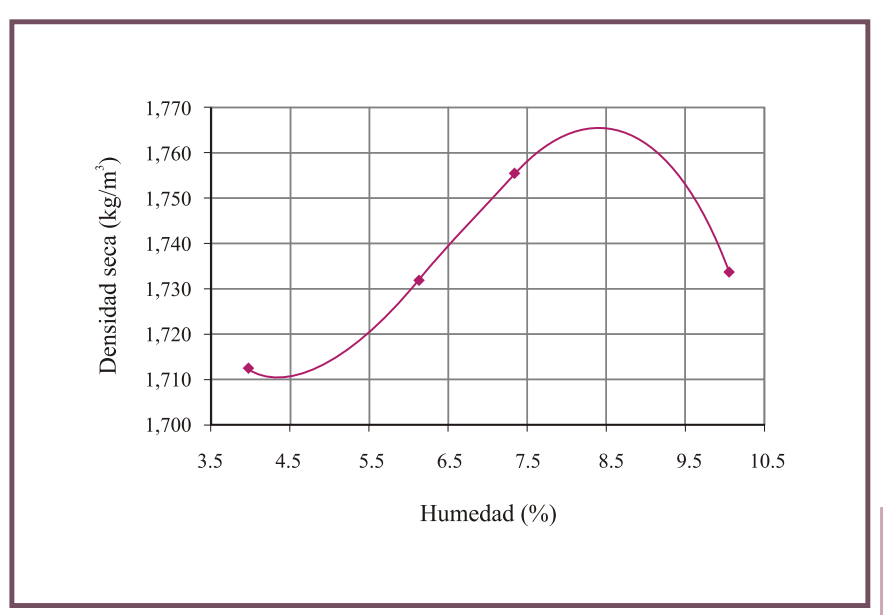

Figura 2. Curva de compactación de la arena.

La necesidad de utilizar como refuerzo telas, en lugar de los geotextiles fabricados por la empresa, se debió a las limitaciones de carga que presenta la máquina construida, ya que los geotextiles cuentan con altas resistencias, y de haberlos usado en los modelos, difícilmente se hubiera logrado la falla del suelo reforzado. Los ensayos realizados a las telas se presentan en la tabla 2; en la figura 3 se muestran los testigos de tela de interlón utilizados en los ensayos adelantados en la empresa Pavco.

En la tabla 3 se presentan los resultados obtenidos en las pruebas realizadas en Pavco para la tela de interlón; se presentan solo estos debido a que esta tela fue la utilizada en la construcción de los modelos. 
Tabla 2. Pruebas realizadas a las telas.

\begin{tabular}{|l|c|c|}
\hline Parámetro & Norma & $\begin{array}{c}\text { N. }{ }^{\circ} \text { de probetas } \\
\text { por tela }\end{array}$ \\
\hline Resistencia a la tensión & NTC 3334-92 & 2 \\
\hline Carga de ruptura y elongación & NTC1998-83 & 4 \\
\hline Resistencia al desgaste trapezoidal & NTC 2003-84 & 2 \\
\hline Masa por unidad de área & NTC1995-85 & 1 \\
\hline Resistencia a la ruptura & & 1 \\
\hline
\end{tabular}

Nota: Ensayos realizados en Pavco.

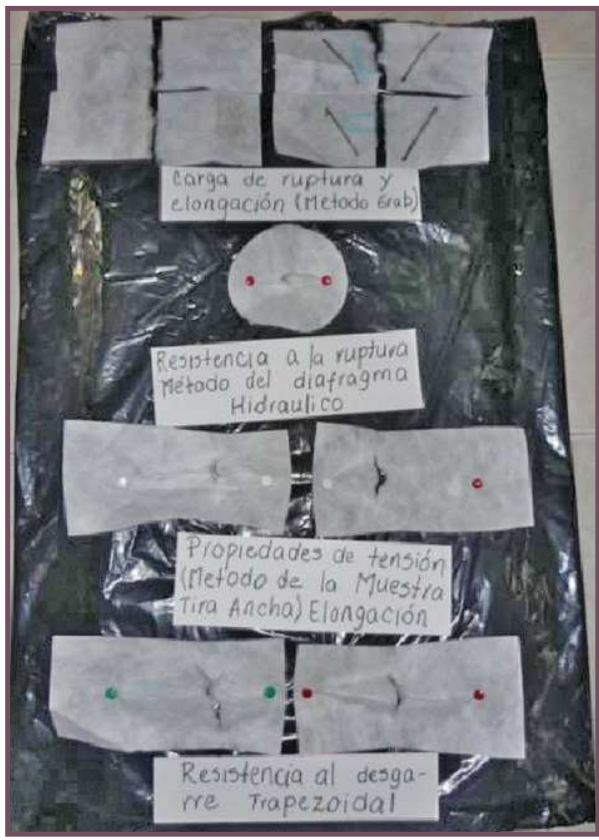

Figura 3. Testigos de tela interlón para los ensayos de laboratorio realizados en la empresa Pavco.

Tabla 3. Propiedades de la tela de interlón.

\begin{tabular}{|l|c|c|}
\hline Parámetro & Unidad & $\begin{array}{c}\text { Valor promedio } \\
* \mathrm{~L} / \mathrm{T}\end{array}$ \\
\hline Resistencia a la tensión/Elongación & $\begin{array}{c}\mathrm{kN} / \mathrm{m} \\
\%\end{array}$ & $\begin{array}{c}2.18 / 1.67 \\
12 / 18\end{array}$ \\
\hline Carga de ruptura y elongación & $\begin{array}{c}\mathrm{N} \\
\%\end{array}$ & $\begin{array}{c}143 / 154 \\
21 / 33\end{array}$ \\
\hline Resistencia al desgaste trapezoidal & $\mathrm{N}$ & $21 / 25$ \\
\hline Masa por unidad de área & $\mathrm{g} / \mathrm{m}^{2}$ & 78.5 \\
\hline Resistencia a la ruptura & $\mathrm{psi}$ & 65 \\
\hline
\end{tabular}

*L/T: Sentido longitudinal/Sentido transversal.

\section{DESCRIPCIÓN DEL EQUIPO}

La máquina diseñada y construida para el desarrollo del proyecto está conformada por una estructura metálica de dos pórticos, los cuales se encuentran unidos mediante pernos de alta resistencia. Estos dos soportes están compuestos por vigas en perfil en canal de 4 pulgadas de alto con un espesor de $1 / 4$ de pulgada y columnas en ángulos de lados iguales de $2 \frac{1}{2}$ " y $\mathrm{e}=1 / 4$ ", los cuales se atornillan conformando una estructura rígida. La máquina tiene una longitud de $1,20 \mathrm{~m}$, una altura de $1,40 \mathrm{~m}$ y anchos que pueden variar desde $0,20 \mathrm{~m}$ hasta $0,60 \mathrm{~m}$. Adicionalmente a este sistema de pórticos, se encuentran dispuestos un número importante de elementos que trabajan conjuntamente y que permiten la realización de las pruebas.

Dentro de los principales sistemas que conforman la máquina se presentan los de aplicación de carga vertical, carros móviles, caja para disposición de modelos y de medición de cargas y desplazamientos.

El sistema de aplicación de carga es un conjunto de mecanismos de poleas y engranajes, que trabajan coordinadamente hasta lograr desplazar un pistón que aplica la fuerza de manera continua; éste es accionado por un motor eléctrico de $1 \mathrm{hp} \mathrm{de}$ potencia, que permite realizar inversiones de giro según las necesidades. La carga puede ser aplicada en cualquier parte del modelo, ya que el equipo cuenta con un sistema de carros móviles que se desplazan tanto en sentido longitudinal como ortogonal a las vigas principales, permitiendo colocar una placa de apoyo de dimensiones variadas.

La adquisición de datos de cargas y desplazamientos del modelo se realiza con técnicas tanto digitales como manuales. Para la medición de la carga, la máquina cuenta con una celda digital con indicador de $1 \mathrm{~kg}$-f de precisión y capacidad de $5000 \mathrm{~kg}$-f (ver figura 4). Los desplazamientos verticales experimentados por la placa de carga se registran con una regleta graduada con divisiones al milímetro.

En el modelo es posible conocer en forma aproximada los desplazamientos tanto horizontales como verticales, que pueden generarse en las capas del modelo, gracias al sistema de coordenadas que se tiene dispuesto en el acrílico y en el suelo. Este sistema de referenciación funciona, ya que uno de ellos permanece fijo mientras que el localizado en el suelo se mueve con la aplicación de carga.

\section{MODELACIÓN FÍSICA}

\subsection{Características generales de los modelos}

La maquina construida permite elaborar modelos a escala reducida de muros en tierra armada con geotextil, y someterlos a un proceso de carga de compresión. El modelo propuesto presenta limitaciones propias de los modelos a escala reducida, debido a que no se cuenta con máquina centrífuga para garantizar que los esfuerzos desarrollados en el modelo sean 
Representativos de los que experimenta el prototipo (Rodríguez y Rincón, 2001). Sin embargo, el montaje construido permite obtener la curva esfuerzo-desplazamiento de los modelos y estudiar el mecanismo de falla que presentan este tipo de estructuras, considerando esto un aporte importante al estudio de estructuras en tierra armada.

Teniendo presente las recomendaciones dadas por el ingeniero Jaime Suárez (Suárez, 2004) en el diseño y construcción de muros en suelo reforzado con geosintéticos, y descubriendo que algunas de ellas pueden adaptarse a la conformación de modelos, los autores diseñaron tres tipos de muros experimentales sobre los cuales se basó la investigación. Los modelos consisten en un muro de $0,60 \mathrm{~m}$ de alto, $0,80 \mathrm{~m}$ de largo y $0,20 \mathrm{~m}$ de ancho de arena reforzada con tela de interlón; en total se realizaron 6 modelos con diferentes espesores de capa reforzada $(10 \mathrm{~cm}, 15 \mathrm{~cm}$ y $20 \mathrm{~cm})$, dos de cada uno en condiciones similares de humedad y densidad. En la tabla 4 se presentan las características geométricas y constructivas de cada uno de los modelos realizados.

Tabla 4. Características geométricas y constructivas para la conformación de los modelos.

\begin{tabular}{|l|c|c|c|}
\hline Parámetro & $\begin{array}{c}\text { Modelos } \\
\text { tipo 1 } \\
\text { (A y B) }\end{array}$ & $\begin{array}{c}\text { Modelos } \\
\text { tipo 2 } \\
\text { (A y B) }\end{array}$ & $\begin{array}{c}\text { Modelos } \\
\text { tipo 3 } \\
\text { (A y B) }\end{array}$ \\
\hline Vel. de aplicación de la carga (mm/min) & 18,1 & 18,1 & 18,1 \\
\hline Altura del modelo (cm) & 60,0 & 60,0 & 60,0 \\
\hline Ancho de placa (cm) & 20,0 & 20,0 & 20,0 \\
\hline Longitud del modelo (cm) & 80,0 & 80,0 & 80,0 \\
\hline Longitud del refuerzo (cm) & 68,0 & 68,0 & 68,0 \\
\hline Longitud de anclaje Ll (cm) & 15,0 & 15,0 & 15,0 \\
\hline Esp. vertical entre capas Sv (cm) & 10,0 & 15,0 & 20,0 \\
\hline N. ${ }^{\circ}$ de golpes por subcapa de 5 cm & 160 & 160 & 160 \\
\hline N. ${ }^{\circ}$ de capas de reforzadas & 6 & 4 & 3 \\
\hline
\end{tabular}

Nota: Se ensayaron 6 modelos, dos para cada espesor de capa reforzada.

\subsection{Descripción del proceso constructivo de los modelos}

Previo a la construcción de los modelos, se definieron sus características geométricas y constructivas como: número de capas, condiciones de humedad, número de golpes, entre otros. A continuación se describe el proceso constructivo de los modelos:

- Verificación de condiciones de humedad de la arena. Se define si es necesario añadir o retirar agua de la arena, para alcanzar una humedad próxima a su óptima.

- Definición de sistemas de coordenadas (x,y) en el acrílico, las coordenadas marcadas en el refuerzo del modelo deben coincidir con estas para realizar un control adecuado de los desplazamientos.
- Colocación de formaleta en madera, esta se debe hacer coincidir con alguna de las divisiones del sistema de coordenadas. La formaleta se ajusta y sostiene con ayuda de un gato mecánico.

- Corte de la tela de refuerzo, de acuerdo con las dimensiones del modelo, altura de capa y longitud de empotramiento.

- Colocación de la tela de refuerzo.

- Colocación, distribución y compactación de capas de arena de $5 \mathrm{~cm}$ de espesor hasta conformar el espesor de capa reforzada. A cada capa de $5 \mathrm{~cm}$ se aplica la misma energía de compactación.

- Consecutivamente se continúa con la colocación de refuerzo y compactación de capa de arena, hasta alcanzar la altura de $60 \mathrm{~cm}$.

- Ajuste de la placa de carga e inicio del proceso de carga.

\subsection{Resultados de la modelación}

Los parámetros medidos durante el proceso de falla fueron la carga y el desplazamiento experimentado por el modelo; adicionalmente se observó el mecanismo de falla en cada modelo ensayado. A continuación se presentan los resultados obtenidos en el proceso de carga de los modelos:

- Modelo tipo 1: consiste en un muro de $60 \mathrm{~cm}$ de alto, el espesor de la capa reforzada es igual a $10 \mathrm{~cm}$, para un total de 6 capas (ver tabla 4). En las figuras 4 y 5 se presenta el modelo tipo 1 en la condición inicial del proceso de carga y en la condición final, respectivamente. En la figura 4 se observa el número de capas y el sistema de coordenadas de referencia utilizado. En la figura 5 se observa el mecanismo de falla experimentado por el modelo tipo 1, en esta se muestra la cuña de muro fallado y la rotura del material de refuerzo. La superficie de falla es aproximadamente planar y sale a la pata del muro.

El esfuerzo en la falla promedio experimentado por el modelo tipo 1 fue igual a $3,56 \mathrm{~kg} / \mathrm{cm}^{2}$, y su correspondiente deformación unitaria resultó igual a 7,29\%. En la figura 6 se presenta la curva esfuerzo-deformación del modelo tipo 1 (ver tabla 5); en esta se observa un buen ajuste para los dos modelos ensayados; el inicio de la curva muestra un acomodamiento de la placa sobre el modelo, mientras se hace el contacto adecuado.

Tabla 5. Resultados obtenidos del modelo tipo 1.

\begin{tabular}{|l|c|c|}
\hline Parámetro & $\begin{array}{c}\text { Modelo } \\
\text { 1-A }\end{array}$ & $\begin{array}{c}\text { Modelo } \\
\text { 1-B }\end{array}$ \\
\hline N. ${ }^{\circ}$ de capas con refuerzo & 6 & 6 \\
\hline Esfuerzo de falla (kg/cm2) & 3,57 & 3,55 \\
\hline $\begin{array}{l}\text { Desplazamiento vertical al } \\
\text { punto de falla (cm) }\end{array}$ & 4,25 & 4,50 \\
\hline Deformación unitaria en la falla (\%) & 7,08 & 7,50 \\
\hline
\end{tabular}

Nota: La deformación unitaria fue calculada a partir de la dimensión vertical inicial del modelo $(60 \mathrm{~cm})$. 


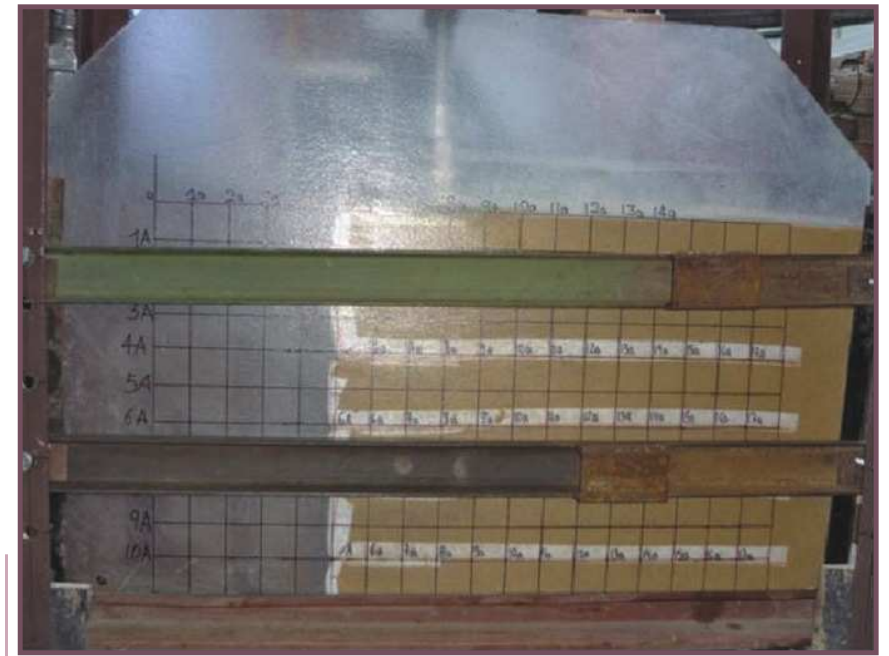

Figura 4. Vista en corte del modelo tipo 1-A

al inicio del proceso del carga.

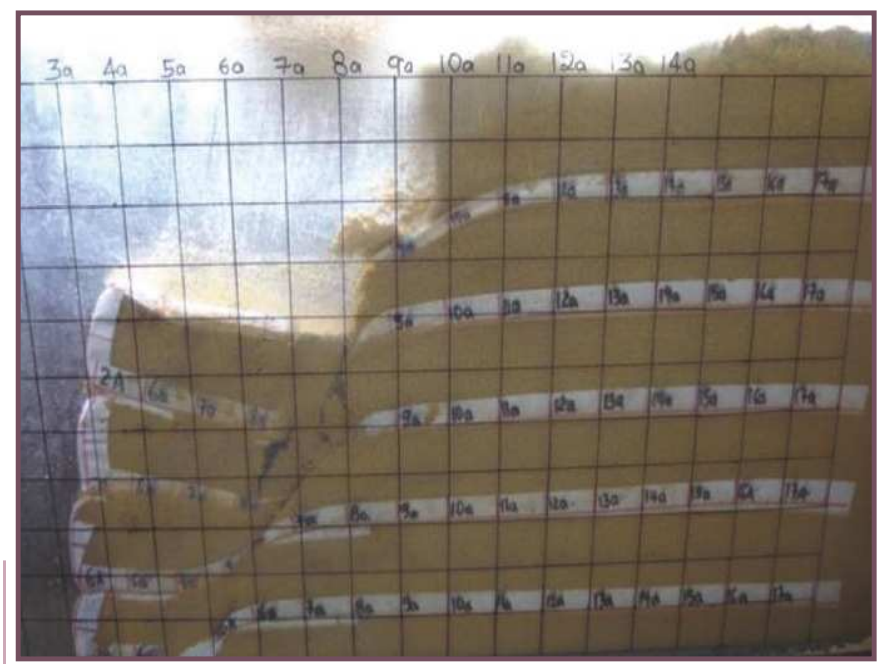

Figura 5. Vista en corte del modelo 1-A

al final del proceso de carga.

- Modelo tipo 2: consiste en un muro de $60 \mathrm{~cm}$ de alto, con un espesor de capa reforzada igual a $15 \mathrm{~cm}$, para un total de 4 capas (ver tabla 6).

El esfuerzo en la falla promedio experimentado por el modelo tipo 2 fue igual a $2,35 \mathrm{~kg} / \mathrm{cm}^{2}$; en el modelo tipo 2 se experimenta una disminución en la máxima resistencia equivalente a $34 \%$ con respecto al modelo tipo 1 . La deformación unitaria en la falla en el modelo 2 es igual a 5,00\%, con respecto al modelo tipo 1; en el modelo tipo 2 se experimenta la falla a un nivel de deformación menor. En la figura 7 se presenta la curva esfuerzo-deformación del modelo tipo 2; en esta se observa un buen ajuste para los dos modelos ensayados; el inicio de la curva del ensayo A muestra un acomodamiento de la placa sobre el modelo mientras se hace el contacto adecuado.

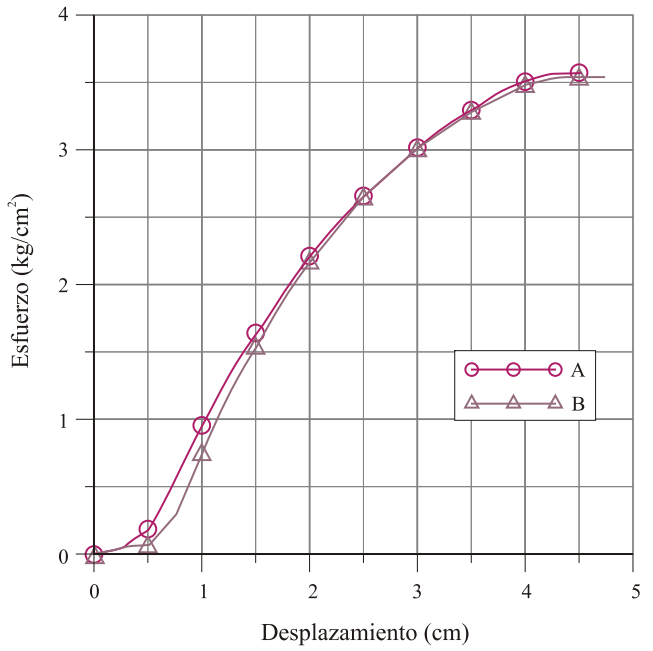

Figura 6. Curva esfuerzo-desplazamiento del modelo tipo 1.

Tabla 6. Resultados obtenidos del modelo tipo 2.

\begin{tabular}{|l|c|c|}
\hline Parámetro & $\begin{array}{c}\text { Modelo } \\
\text { 2-A }\end{array}$ & $\begin{array}{c}\text { Modelo } \\
\text { 2-B }\end{array}$ \\
\hline N. ${ }^{\text {de capas con refuerzo }}$ & 4 & 4 \\
\hline Esfuerzo de falla $\left(\mathrm{kg} / \mathrm{cm}^{2}\right)$ & 2,34 & 2,36 \\
\hline $\begin{array}{l}\text { Desplazamiento vertical al } \\
\text { punto de falla }(\mathrm{cm})\end{array}$ & 3,00 & 3,00 \\
\hline Deformación unitaria en la falla $(\%)$ & 5,00 & 5,00 \\
\hline
\end{tabular}

Nota: La deformación unitaria fue calculada a partir de la dimensión vertical inicial del modelo $(60 \mathrm{~cm})$.

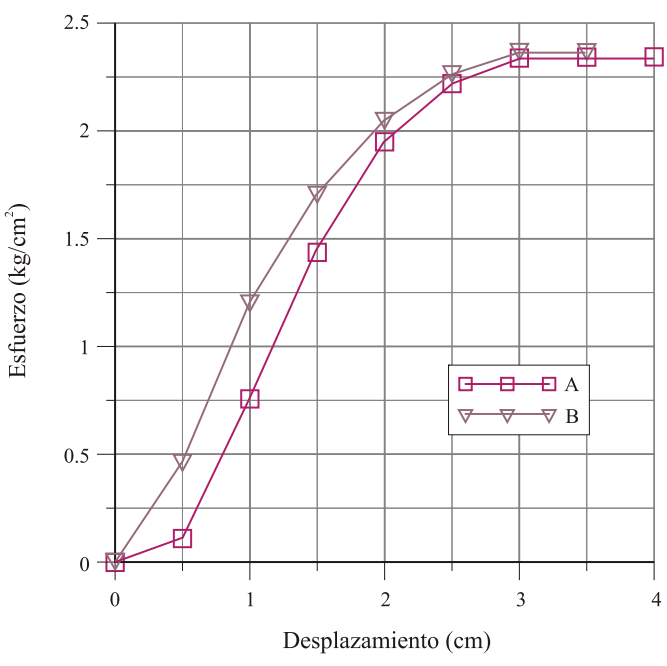

Figura 7. Curva esfuerzo-desplazamiento del modelo tipo 2. 
- Modelo tipo 3: consiste en un muro de $60 \mathrm{~cm}$ de alto, con un espesor de capa reforzada igual a $20 \mathrm{~cm}$, para un total de 3 capas (ver tabla 7).

Tabla 7. Resultados obtenidos del modelo tipo 3.

\begin{tabular}{|l|c|c|}
\hline Parámetro & $\begin{array}{c}\text { Modelo } \\
\text { 3-A }\end{array}$ & $\begin{array}{c}\text { Modelo } \\
\text { 3-B }\end{array}$ \\
\hline N. ${ }^{\text {de capas con refuerzo }}$ & 3 & 3 \\
\hline Esfuerzo de falla $(\mathrm{kg} / \mathrm{cm} 2)$ & 1,54 & 1,53 \\
\hline $\begin{array}{l}\text { Desplazamiento vertical al } \\
\text { punto de falla (cm) }\end{array}$ & 2,25 & 2,00 \\
\hline Deformación unitaria en la falla (\%) & 3,75 & 3,33 \\
\hline
\end{tabular}

Nota: La deformación unitaria fue calculada a partir de la dimensión vertical inicial del modelo $(60 \mathrm{~cm})$.

El esfuerzo en la falla promedio experimentado por el modelo tipo 3 fue igual a $1,54 \mathrm{~kg} / \mathrm{cm}^{2}$; en el modelo tipo 3 se experimenta una disminución en la máxima resistencia, equivalente a $34,65 \%$ con respecto al modelo tipo 2 , y de $56,87 \%$ con respecto al modelo tipo 1 . La deformación unitaria en la falla para el modelo 3 es igual a $3,54 \%$, con respecto a los modelos tipo 1 y 2 ; en el modelo tipo 3 ocurre la falla a un nivel de deformación más bajo. En la figura 8 se presenta la curva esfuerzo-deformación del modelo tipo 3 , en esta se observa un buen ajuste para los dos modelos ensayados, el inicio de la curva, principalmente del ensayo A, muestra un acomodamiento de la placa sobre el modelo mientras se hace un contacto adecuado. En la tabla 8 se presenta el resumen de resultados promedio de los modelos.

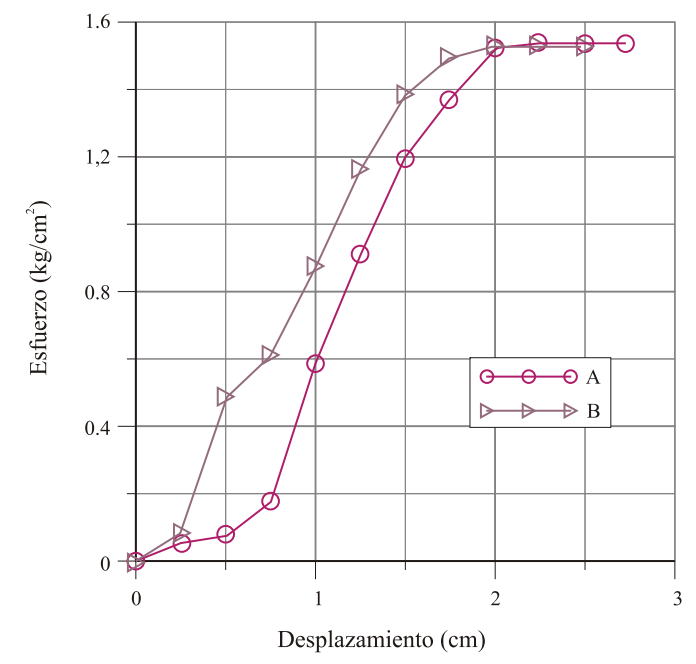

Figura 8. Curva esfuerzo-desplazamiento del modelo tipo 3.
Tabla 8. Resultados promedio de los modelos.

\begin{tabular}{|c|c|c|}
\hline Modelo & $\begin{array}{c}\text { Esfuerzo máximo } \\
\text { en la falla }\left(\mathbf{K g} / \mathbf{c m}^{2}\right)\end{array}$ & $\begin{array}{c}\text { Deformación } \\
\text { unitaria (\%) }\end{array}$ \\
\hline 1 & 3,56 & 7,29 \\
\hline 2 & 2,35 & 5,00 \\
\hline 3 & 1,54 & 3,54 \\
\hline
\end{tabular}

Nota: la deformación unitaria fue calculada a partir de la dimensión vertical inicial del modelo $(60 \mathrm{~cm})$.

Al realizar una comparación entre el número de capas de refuerzo y el esfuerzo máximo, se observa una correlación acentuada (ver figura 9). Estos resultados presentan una relación lineal e indican la proporcionalidad entre las variables consideradas; lo anterior permite establecer tendencias en el comportamiento de muros de contención en tierra reforzada con geotextil, sin embargo, será importante realizar mayor cantidad de ensayos para obtener expresiones para otros tipos de materiales y diferentes geometrías de muros.

En la tabla 9 se presenta la inclinación aproximada de la superficie de falla de los seis modelos ensayados; en general, la superficie de falla se ajusta a una planar; en algunos modelos el plano de falla no salió a la pata del muro, sino por encima de la primera capa, que puede considerarse como una tendencia en el comportamiento; sin embargo, no se tiene claro si se debe al hecho de que el modelo se construye sobre un material de una rigidez mucho mayor que el material del modelo. El ángulo de fricción de la arena es igual a $33^{\circ}$; de acuerdo con lo consultado en la bibliografía (Das, 1999), se espera que la inclinación de la superficie de falla sea igual a $45+\phi / 2$, con respecto a la horizontal, en este caso resultaría igual a $61,5^{\circ}$. Al analizar los resultados de la tabla 9 , se observa un ajuste satisfactorio para algunos de los modelos (1-A, 2-A, 2-B). El equipo construido permitirá profundizar en la validación de esta propuesta de inclinación de superficie de falla, con la ejecución de más ensayos y utilización de diferentes tipos de suelo.

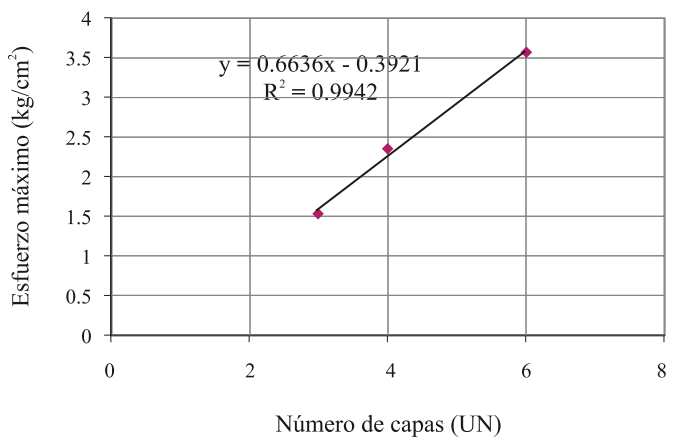

Figura 9. Correlación entre el número de capas de refuerzo y el máximo esfuerzo. 
Tabla 9. Inclinación aproximada de la superficie de falla de la cuña.

\begin{tabular}{|c|c|}
\hline Modelo & $\begin{array}{c}\text { Inclinación aproximada } \\
\text { de la superficie de falla }\left(^{\circ}\right)\end{array}$ \\
\hline $1-A$ & 61,21 \\
\hline $1-B$ & 53,57 \\
\hline 2-A & 59,51 \\
\hline 2-B & 62,09 \\
\hline $3-A$ & 53,57 \\
\hline 3-B & 57,52 \\
\hline
\end{tabular}

\section{CONCLUSIONES}

Se comprobó que los resultados entre ensayos con las mismas características de construcción no presentaron variaciones mayores al $2 \%$.

Se verificó que entre mayor número de capas de refuerzo utilizadas en los modelos, mayor es la deformabilidad del muro antes de alcanzar la falla.

A pesar de que se planteó un procedimiento constructivo para la conformación de los modelos, este puede estar sujeto a ajustes, los cuales se logran con la experiencia y la disponibilidad de equipos de pesaje y de medición, y con la realización de un mayor número de ensayos.

Con base en los resultados encontrados durante la realización de las pruebas se puede observar una correlación directa entre la resistencia de los muros y el número de capas. Esta se ajustó a un comportamiento de tipo exponencial; sin embargo, sería conveniente un mayor número de pruebas para confrontar esta tendencia.

Gracias al sistema de coordenadas de tipo móvil y fijo es factible observar las diferentes trayectorias que van presen- tando las telas durante el desarrollo de las pruebas. Por lo tanto, se pueden apreciar las altas deformaciones de tipo vertical y horizontal que alcanzaron a ser, incluso, mayores al $7 \%$ antes de que se genere la falla en los modelos.

Con la realización de los modelos se pueden apreciar plenamente los mecanismos de falla que experimentan los muros ensayados, lo cual permite predecir su comportamiento.

Debido a las características presentadas en los refuerzos después de generada la falla, se puede estimar la distribución del esfuerzo a medida que se aumenta la profundidad.

El equipo construido permitirá profundizar en la validación de teorías que procuran determinar la inclinación de la superficie de falla para estructuras de tierra armada con geotextil.

Con base en los resultados encontrados durante la realización de las pruebas, se puede observar una correlación directa entre la resistencia de los muros y el número de capas. Esta se ajustó a un comportamiento de tipo exponencial; sin embargo, sería conveniente un mayor número de pruebas para confrontar esta tendencia.

\section{REFERENCIAS}

Centeno, F. (1985). Ingeniería biotécnica y bioingeniería de suelos. XVII Seminario venezolano de geotecnia, Caracas.

Das, B. (1999). Principios de ingeniería de cimentaciones. California: Thompson.

Holtz, R. y Lee, W. (2002). Internal Stability Analyses of Geosynthetic Reinforced Retaining Walls. Research Report. Agreement No. T9903, Task 95. Washington D.C.: Washington State Transportation Center.

López, C. (1999). Manual de estabilización y revegetación de taludes. Madrid: Entorno Gráfico, S.L.

Rodríguez, E. y Rincón, C. (2001). Modelación física en centrífuga de excavación sostenida con muro pantalla. Tesis de Maestría en Ingeniería Civil, Departamento de Ingeniería Civil y Ambiental. Universidad de los Andes.

Suárez, J. (2004). Diseño de muros de suelo reforzados con geosintéticos. Bucaramanga: Publicaciones UIS. 